\title{
The Effect of Model Fidelity on Prediction of Char Burnout for Single-Particle Coal Combustion
}

\author{
Josh McConnell ${ }^{\mathrm{a}}$, James C. Sutherland ${ }^{\mathrm{a}, *}$ \\ ${ }^{a}$ Department of Chemical Engineering, \\ University of Utah, Salt Lake City, UT
}

\begin{abstract}
Practical simulation of industrial-scale coal combustion relies on the ability to accurately capture the dynamics of coal subprocesses while also ensuring the computational cost remains reasonable. The majority of the residence time occurs post-devolatilization, so it is of great importance that a balance between the computational efficiency and accuracy of char combustion models is carefully considered. In this work, we consider the importance of model fidelity during char combustion by comparing combinations of simple and complex gas and particle-phase chemistry models. Detailed kinetics based on the GRI 3.0 mechanism and infinitely-fast chemistry are considered in the gasphase. The Char Conversion Kinetics model and $n^{\text {th }}$-Order Langmuir-Hinshelwood model are considered for char consumption. For devolatilization, the Chemical Percolation and Devolatilization and Kobayashi-Sarofim models are employed. The relative importance of gasification versus oxidation reactions in air and oxyfuel environments is also examined for various coal types. Results are compared to previously published experimental data collected under laminar, single-particle conditions. Calculated particle temperature histories are strongly dependent on the choice of gas phase and char chemistry models, but only weakly dependent on the chosen devolatilization model. Particle mass calculations were found to be very sensitive to the choice of devolatilization model, but only somewhat sensitive to the choice of gas chemistry and char chemistry models. High-fidelity models for devolatilization generally resulted in particle temperature and mass calculations that were closer to experimentally observed values.
\end{abstract}

\section{Introduction}

Char oxidation and gasification are complex phenomena complicated by poorly understood heterogeneous reaction mechanisms and an evolving morphology that has a strong influence on the transport properties within a reacting char particle. Additionally, turbulent flow in industrial coal combustion systems adds significant complexity due to the nonlinear coupling that occurs across the additional length and timescales introduced. These factors, along with the consideration that computational resources are finite, pose a significant challenge for modeling industrial-scale coal boilers. Coal combustion simulations often use empirical models in place of more complex physical approaches to mitigate the computational burden. Therefore, it is important to know which phenomena need to be resolved to balance computational cost with accuracy. Acquiring this knowledge for char combustion is especially important, as most of the residence time occurs post-devolatilization.

${ }^{*}$ Corresponding Author

Email address: James.Sutherland@utah.edu (James C. Sutherland) 
Several approaches have been taken to model char combustion and gasification, ranging from models that only consider the bulk partial pressure of major reacting species [1] to more complex models that rely on calculation of surface pressures of reactants and incorporate the effects of evolving char properties [2, 3, 4, 5. One of the first models to consider the intricacies of char reaction kinetics is the Char Burnout Kinetics (CBK) model developed by Hurt and others [4], which incorporates multistep oxidation [2] and gasification by $\mathrm{CO}_{2}, \mathrm{H}_{2} \mathrm{O}$, and $\mathrm{H}_{2}$ [3] as well as intraparticle species gradients. The Char Conversion Kinetics (CCK) model developed by Shurtz and Fletcher [5, 6] combines the char oxidation and gasification submodels of [2] and [3] and adds consideration of Stefan flow.

Several recent studies have been undertaken to assess simplifying assumptions typically used in modeling char combustion and gasification. Gonzalo-Tirado et. al. 7] compared several approaches to modeling CO oxidation around a burning char particle and demonstrate that the choice of gas-phase chemistry has a significant impact on the calculated particle temperature. A study by Hecht et. al. 8] examines the implementation of single, double, and continuous-film models for modeling char combustion utilizing either intrinsic or apparent surface kinetics, and shows that predicted temperature and char consumption rates are strongly dependent on the film model and weakly dependent on the choice of apparent or intrinsic heterogeneous kinetics. Very little work appears to have been done in comparing char consumption models when coupled with both devolatilization and gas phase chemistry. Recent work has compared the performance of combinations of high and low fidelity devolatilization and gas phase chemistry models in the context of ignition delay for laminar single particle studies [9] as well as flame standoff for turbulent combustion [10], but focus on devolatilization rather than char burnout.

The goal of this work is to examine the influence of model fidelity in particle and gas-phase chemistry models on the char oxidation process and determine which aspects of coal combustion need to be modeled with high fidelity in order to accurately predict quantities of interest. To accomplish this, we perform simulations with various combinations of devolatilization, char consumption (both gasification and oxidation), and gas phase chemistry models and compare the results to experimentally collected data for particle mass and temperature for two coal types: a high rank bituminous coal (Eastern Bituminous) and a low rank sub-bituminous coal (Highvale). In the gas phase, kinetics based on the GRI 3.0 mechanism [11] and infinitely fast chemistry are utilized. For devolatilization, the Chemical Percolation and Devolatilization (CPD) and Kobayashi-Sarofim (KS) models are considered. For char consumption, the CCK model and an $n^{\text {th }}$-order Langmuir-Hinshelwood (LH) oxidation model developed by Murphy and Shaddix [12] coupled with first-order Arrhenius expressions for gasification are employed.

\section{Theoretical Formulation}

A time-evolving one-dimensional domain along the $x$-coordinate was used in this work. The set of equations given in the following sections closely follows the formulations provided in [9, 10.

\subsection{Gas Phase}

The gas phase Conservation equations are written in an Eulerian reference frame [9, 10]

$$
\frac{\partial \rho \phi}{\partial t}=-\frac{\partial \rho \phi u}{\partial x}-\frac{\partial \Theta_{\phi}}{\partial x}+\omega_{\phi}+\sum_{j=1}^{n_{\mathrm{p}}} \frac{S_{\mathrm{p}_{j} \phi}}{V_{\text {cell }}},
$$


where $\phi$ is an intensive quantity, $\Theta_{\phi}$ is the diffusive flux of $\phi, \omega_{\phi}$ is the net rate of production of $\phi$ in the gas phase, $V_{\text {cell }}$ is the quantity representing the volume of the control volume, and $S_{\mathrm{p}_{j} \phi}$ is the particle to gas source of $\phi$. In this formulation, $\phi=\left\{1, \rho u, \rho v, \rho e_{0}, \rho Y_{i}\right\}$ where $\rho$ is the mass density, $u$ and $v$ are the $x$ and $y$ components of velocity, respectively, $e_{0}$ is the specific total internal energy, and $Y_{i}$ are species mass fractions. For continuity, $\phi=1$ and $\Theta_{\rho}=0$. The energy source term due to radiative heat transfer from the gas to the surroundings assumes a grey and optically thin gas: $S_{\mathrm{rad}}=-\kappa_{\mathrm{g}} \sigma\left(T_{\mathrm{g}}^{4}-T_{\mathrm{w}}^{4}\right)$, where $\kappa_{\mathrm{g}}$ and $T_{\mathrm{g}}$ are the absorption coefficient and temperature of the gas, respectively. The gas absorption coefficient was determined by summing contributions from tar, soot, $\mathrm{H}_{2} \mathrm{O}$, and $\mathrm{CO}_{2}$. The absorption coefficients for tar and soot were determined from an expression in [13. Absorption coefficients for $\mathrm{H}_{2} \mathrm{O}$, and $\mathrm{CO}_{2}$ were taken from [14].

\subsection{Particle Phase}

Particles are transported in a Lagrangian reference frame where the position, velocity, mass, temperature, and composition of individual particles are evolved in time and are two-way coupled to the gas phase. Gas displacement by the particle is neglected. Further details are available in [9, 10].

\subsection{Interphase Coupling}

The exchange term for each species is calculated by summing contributions from devolatilization, evaporation, char oxidation, and char gasification. Terms for evaporation and devolatilization are given in [9], while models for oxidation and gasification will be discussed in $\$ 2.5 .1,2.5 .2$.

The quantity $\alpha=0.3$ is the fraction of heat due to heterogeneous reactions evolved to the gas phase, with $1-\alpha$ the fraction of heat absorbed by the particle.

$$
\Delta H^{\mathrm{Ox}}=\frac{\varphi \Delta H_{\mathrm{CO}_{2}}^{\mathrm{Ox}}+\Delta H_{\mathrm{CO}}^{\mathrm{Ox}}}{1+\varphi},
$$

where $\varphi$ is the instantaneous ratio of $\mathrm{CO}_{2}$ to $\mathrm{CO}$ produced through char oxidation. The source term in the particle energy balance is given as

$$
\begin{array}{r}
S_{\mathrm{r}}=\frac{1-\alpha}{m_{\mathrm{p}} C_{\mathrm{p}}}\left[\Delta H^{\text {Evap }}\left(\frac{\mathrm{d} m_{\mathrm{p}}}{\mathrm{d} t}\right)^{\text {Evap }}\right. \\
\left.+\Delta H^{\mathrm{Ox}}\left(\frac{\mathrm{d} m_{\mathrm{c}}}{\mathrm{d} t}\right)^{\mathrm{Ox}}+\sum_{k} \Delta H_{k}^{\mathrm{G}}\left(\frac{\mathrm{d} m_{\mathrm{c}}}{\mathrm{d} t}\right)_{k}^{\mathrm{G}}\right]
\end{array}
$$

where $m_{\mathrm{p}}$ is the particle mass, $m_{\mathrm{c}}$ is the mass of char within the particle, and $k=\left\{\mathrm{CO}_{2}, \mathrm{H}_{2} \mathrm{O}, \mathrm{H}_{2}\right\}$. The superscripts "Evap", "Ox" and "G" denote evaporation, oxidation, and gasification, respectively,

\subsection{Gas Phase Chemistry}

Two models for gas phase chemistry were used in this work: detailed kinetics based on GRI 3.0 [1] and infinitelyfast (flame-sheet) chemistry (see [10]). In both of the models to be described, transport equations for each species, with the corresponding phase exchange terms for char oxidation and gasification, are solved at each point in time and space.

A model for reactions involving tar and soot developed by Brown and Fletcher [15] is utilized for both detailed kinetics and flame-sheet models. Products of tar and soot oxidation are assumed to be $\mathrm{CO}$ and $\mathrm{H}_{2} \mathrm{O}$. 


\subsection{Particle Phase Chemistry}

Proper modeling of coal combustion requires accounting for evaporation, loss of volatiles, and char consumption though oxidation and gasification. The Chemical Percolation and Devolatilization (CPD) model [16] is used because of its ability to accurately predict volatile yields with speciation. This study compares the effect of the two-step Kobayashi-Sarofim [17] and CPD devolatilization models on char burnout. Kinetic parameters reported in [18] are used with the two-step model.

Char oxidation and gasification depend on intrinsic char characteristics including the pore structure, carbon crystallinity, and ash content which have a significant influence on intraparticle gradients of gaseous reactants and the reactivity of the char. The CCK model [5, described in 2.5.1] attempts to account for these effects. Other

models, such as the $n^{\text {th }}$-order Langmuir-Hinshelwood model [12] described in $\$ 2.5 .2$ forgo this approach and absorb the detailed physics into empirical parameters.

\subsubsection{CCK Model}

The CCK model considers oxidation as well as gasification by $\mathrm{CO}_{2}, \mathrm{H}_{2} \mathrm{O}$, and $\mathrm{H}_{2}$ in 2 reversible and 6 irreversible reactions [5]. Forward and reverse rate constants corresponding to each of the reactions are defined by $k_{j}=f_{\mathrm{RPM}} f_{\mathrm{T}} A_{j, 0} \exp \left(-E_{j} / R T_{p}\right)$, where $f_{\mathrm{RPM}}$ is a factor accounting for internal surface area evolution using a random pore model given in [19, $f_{T}$ is the thermal annealing factor which accounts for the thermal deactivation of the char. Arrhenius parameters for each reaction are determined using correlations in 2, 3]. For zone II or III combustion/gasification, the thermal annealing factor is calculated by integrating the instantaneous fraction of active sites, $\theta$, over a distribution of activation energies, $E_{\mathrm{d}}$, [4]

$$
f_{\mathrm{T}}=\left(\int_{0}^{\infty} \theta d E_{\mathrm{d}}\right)^{1 / 2}
$$

$\theta\left(E_{\mathrm{d}}\right)$ evolves in time according to $\partial \theta / \partial t=-f_{\text {Dev }} A_{\mathrm{d}} \theta \exp \left(-E_{\mathrm{d}} / R T_{\mathrm{p}}\right)$, where $A_{\mathrm{d}}$ is the annealing frequency factor and $f_{\mathrm{Dev}}$ is a correction factor couping the thermal annealing model to devolatilization given by

$$
f_{\text {Dev }}=\exp \left(-a\left(m_{\mathrm{v}} / m_{\mathrm{v}, 0}-m_{\mathrm{v}}\right)\right)
$$

where $m_{\mathrm{v}, 0}$ is the initial mass of volatiles within the coal particle. The function of $f_{\mathrm{Dev}}$ is to inhibit the decay of $f_{\mathrm{T}}$ before and during devolatilization. Without $f_{\mathrm{Dev}}$, the reactivity of the char (and char consumption rate) is underestimated. In this study, $a=30$ and $\theta$ is initialized to a lognormal distribution in $E_{\mathrm{d}}$, with parameters as suggested in [4. An effectiveness factor for each species reaction rate was utilized to account for intraparticle concentration gradients within the char particle. A detailed formulation of expressions for surface reaction rates for each species is given by [2], [3], and [5].

Intraparticle species transport employs a single film model with consideration of a growing ash film [6]. Because each reaction rate depends on one or more of the species partial pressures at the particle surface, the following expression must be solved for each species

$$
P_{i, \mathrm{~s}}=\left\{\begin{array}{cc}
\frac{P}{\gamma_{i}}\left[1-\left(1-\gamma_{i} \frac{P_{i}}{P}\right)\right. & \\
\left.\times \exp \left(-\frac{\gamma_{i} q_{i} R T_{m}}{h_{i} P}\right)\right] & \gamma_{i} \neq 0 \\
P_{i}+\frac{q_{i} R T_{m}}{h_{i}} & \text { otherwise }
\end{array}\right.
$$


where $P_{i}^{\mathrm{s}}$ and $P_{i}$ are the surface and bulk partial pressures of species, $i$, respectively, $\gamma_{i}$ is the volume expansion factor for species $i$ accounting for Stefan flow, $q_{i}$ is the production rate of species $i$ per external particle surface area, $T_{m}$ is the arithmetic mean of the particle and interpolated gas temperatures, and $h_{i}$ is the mass transfer coefficient for species $i$ calculated using the method described in [20. Mode-of-burning relations used to relate time-evolving particle density and mass and expressions for ash film thickness and porosity are taken from [4]. The species source terms due to heterogeneous reactions are calculated as $\left(\mathrm{d} m_{\mathrm{c}} / \mathrm{d} t\right)_{i}=\pi d_{\mathrm{p}}^{2} M_{i} q_{i}$, where $i=\left\{\mathrm{CO}_{2}, \mathrm{CO}, \mathrm{O}_{2}, \mathrm{H}_{2}, \mathrm{H}_{2} \mathrm{O}, \mathrm{CH}_{4}\right\}$ and $M_{i}$ is the molecular weight of species $i$.

The overall char consumption rate is given as

$$
\frac{\mathrm{d} m_{\mathrm{c}}}{\mathrm{d} t}=\left(\frac{\mathrm{d} m_{\mathrm{c}}}{\mathrm{d} t}\right)^{\mathrm{Ox}}-\sum_{j} \frac{M_{\mathrm{c}}}{\nu_{j}^{\mathrm{G}} M_{j}}\left(\frac{\mathrm{d} m_{j}}{\mathrm{~d} t}\right)^{G},
$$

where $j=\left\{\mathrm{CO}_{2}, \mathrm{H}_{2} \mathrm{O}, H_{2}\right\}, m j$ is the mass of species $\mathrm{j}$, and $\nu_{\mathrm{i}}$ denotes the moles of species $i$ produced per mole of char reacted with species $i$. The ratio of $\mathrm{CO}_{2}$ to $\mathrm{CO}$ production in equation (2) is obtained directly from information available in CCK as $\varphi=k_{1} c_{\mathrm{O}_{2}, \mathrm{~s}} / k_{2}$, where $c_{\mathrm{O}_{2}, \mathrm{~s}}$ is the concentration of $\mathrm{O}_{2}$ at the char surface and $k_{1}$ and $k_{2}$ are the rate constants corresponding to the rates at which $\mathrm{CO}_{2}$ and $\mathrm{CO}$ are formed at the char surface, respectively.

\subsection{2. $n^{\text {th }}$-Order Langmuir-Hinshelwood}

As a complement to CCK, the two-step $n^{\text {th }}$-order Langmuir-Hinshelwood (LH) model developed in [12] was implemented for char oxidation calculations: $q_{\mathrm{O}_{2}}=k_{2} k_{1} P_{\mathrm{O}_{2}, \mathrm{~s}}^{n} /\left(k_{1} P_{\mathrm{O}_{2}, \mathrm{~s}}^{n}+k_{2}\right)$, where $q_{\mathrm{O}_{2}}$ is the consumption rate of $\mathrm{O}_{2}$ per external surface area of the particle. The ratio of $\mathrm{CO}_{2}$ to $\mathrm{CO}$ used in $\mathrm{LH}$ is modeled as [21] $\varphi=$ $0.02 P_{\mathrm{O}_{2}, \mathrm{~s}}^{0.21} \exp \left(-3070 / T_{\mathrm{p}}\right)$, with $T$ in $\mathrm{K}$ and $P_{\mathrm{O}_{2}, s}$ in atm. Char gasification by $\mathrm{O}_{2}$ and $\mathrm{CO}_{2}$ is accounted for using first-order Arrhenius kinetics $q_{j}^{\mathrm{G}}=P_{j, \mathrm{~s}} A_{j} \exp \left(-E_{j} / R T_{\mathrm{p}}\right)$ with $j=\left\{\mathrm{CO}_{2}, \mathrm{H}_{2} \mathrm{O}\right\}$ is the production rate of species $j$ by gasification. The Arrhenius parameters used for oxidation and gasification are obtained from [12] and [6, 22], respectively. Species mass production rates and the mass of char lost through oxidation are calculated in the manner described in section 2.5 .1 . The overall char consumption was calculated using (7) with $j=\left\{\mathrm{CO}_{2}, \mathrm{H}_{2} \mathrm{O}\right\}$.

\section{Computational Setup}

The computational configuration emulates the entrained-flow laminar reactor where single particle combustion was examined, as described in [12]. These data were chosen because they were collected under conditions in which raw coal undergoes both devolatilization and heterogeneous oxidation, which represents combustion within a commercial system more accurately than systems in which only char oxidation occurs.

The governing equations are solved using a fully-coupled scheme with a second order spatial discretization, explicit time integration, and characteristic boundary conditions [23. The computational domain is $1.4 \mathrm{~cm}$ in length with a grid spacing of $140 \mu \mathrm{m}$ and a time-step of $20 \mathrm{~ns}$. The results presented in this text are grid-converged; calculations performed using finer grid spacings yield the same results for particle temperature and mass. The domain is situated in the $x$-direction and moves in the $y$-direction according to a mean system velocity [24. A single particle is placed at the center of the computational domain. The complete combustion process (devolatilization through char burnout) is considered for a sub-bituminous coal (Highvale) and a bituminous coal (Eastern Bituminous), and four oxygen mole fractions $(0.06,0.12,0.24$, and 0.36$)$. In all cases, mole fractions of $\mathrm{H}_{2} \mathrm{O}$, and $\mathrm{CO}_{2}$ are initialized to 0.14 and 
0.04, respectively, with the balance being $\mathrm{N}_{2}$. The initial particle temperature and density are set at $350 \mathrm{~K}$ and $1200 \mathrm{~kg} / \mathrm{m}^{3}$, respectively, and initial gas temperature is set at $1685 \mathrm{~K}$, consistent with experimental conditions. The temperature of the reactor walls, which is used for radiative heat transfer calculations, was set to $500 \mathrm{~K}$, which is the estimate given in [12]. Unless otherwise specified, the particle diameter was initialized to $115 \mu \mathrm{m}$. This value was chosen because it is the geometric mean of the maximum $(125 \mu \mathrm{m})$ and minimum $(106 \mu \mathrm{m})$ of the particle size distribution used for experiments performed in [12].

\section{Results and Discussion}

We first examine the influence of $\mathrm{O}_{2}$ concentration and coal type on particle temperature and coal mass loss. Two gas phase and particle phase chemistry models and two devolatilization models with differing levels of complexity and computational cost were utilized. Calculations for each set of model combinations (8 in total) are compared to particle temperature $\left(T_{p}\right)$ and mass data.

Figure 1 shows the thermal annealing deactivation factor, $f_{\mathrm{T}}$ and char conversion, $X_{\text {char }}$ for the early stages of char burnout using the annealing model from [4] (solid lines), and a modified form where annealing is attenuated during devolatilization as described in equations (4)-(5). Attenuating the decay of the thermal annealing factor substantially changes the predicted annealing behavior, but does not have a substantial effect on char consumption calculations presented. This suggests that the char consumption rate is diffusion-limited for this situation. However, in kinetically-limited situations, the model for thermal annealing will effect the char consumption rate considerably.

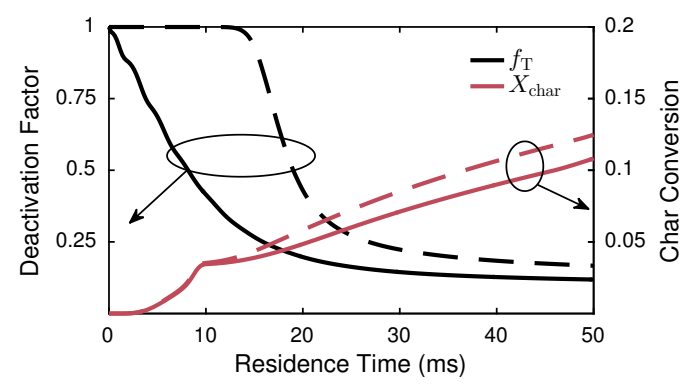

Figure 1: Thermal annealing deactivation factor, $f_{\mathrm{T}}$, and char conversion, $X_{\mathrm{char}}$ in a $6 \% \mathrm{O}_{2}$ environment using the annealing model from [4] unchanged (solid lines), and attenuated during devolatilization as described in equations 44-(5). Calculations utilize flame-sheet, CCK and CPD.

The thermal annealing submodel and mode-of-burning relations within CCK rely a priori specification of the reaction zone in the Three-Zone Theory [25]. A transition from zone II to zone III oxidation behavior is observed in simulations as devolatilization comes to an end, and an additional transition from zone III to zone II occurs as the coal particle approaches complete burnout. Although altering the thermal annealing model during devolatilization had little effect on the char consumption rate in the cases studied, it is presently unknown whether allowing the behavior of the thermal annealing model and mode-of-burning relations to be determined dynamically rather than $a$ priori postulating the combustion mode would result in significantly different particle mass and temperature calculations in post-devolatilization conditions. Furthermore, char deactivation during devolatilization is not well characterized, so it is unclear whether the unaltered char annealing model used within CCK accurately represents thermal deactivation during devolatilization. 
Figure 2 shows predictions of $T_{p}$ and fractional dry ash-free (DAF) mass remaining for $\mathrm{O}_{2}$ mole fractions ranging from $6 \%$ to 36\%, for the Eastern Bituminous coal. Vertical bars that bracket the calculations resulting from detailed gas-phase kinetics indicate the range of values obtained for initial particle diameters ranging from 106-125 $\mu$ m. Ranges for the particle size distribution considered are not shown for calculated $T_{p}$ and DAF particle mass histories resulting from the flame-sheet model since they are very similar to those resulting from detailed gas kinetics calculations. The calculated $T_{p}$ and DAF mass histories for CCK vary more for the range of initial particle diameters considered, as indicated by figure 2. This is likely because the CCK considers an evolving particle diameter and mass transfer resistance due to a growing ash film, whereas the LH model does not. A detailed explanation of the consequences of the aforementioned differences is beyond the scope of this work, but certainly warrants further investigation.

CCK and LH coupled with detailed gas phase kinetics perform similarly well at predicting particle temperature, with CCK having a slight edge in all cases except for the highest $\mathrm{O}_{2}$ concentration considered. Both char models capture the experimental trend for $T_{p}$ quite well for the $6 \%$ and $12 \% \mathrm{O}_{2}$. However, neither of the char models accurately predict $T_{p}$ histories when used with detailed chemistry in the gas phase. Temperature predictions using detailed kinetics in the gas phase are fairly close to experimental values for $\mathrm{O}_{2}$ mole fractions equal to 0.06 and 0.12 independent of the char reaction model used, but predictions for higher $\mathrm{O}_{2}$ concentrations are overestimated by about $300 \mathrm{~K}$. Conversely, use of the flame-sheet model resulted in temperature estimates that greatly underestimated the particle temperature for the lowest two $\mathrm{O}_{2}$ concentrations, but matched experimental values fairly well at the higher oxygen contents.
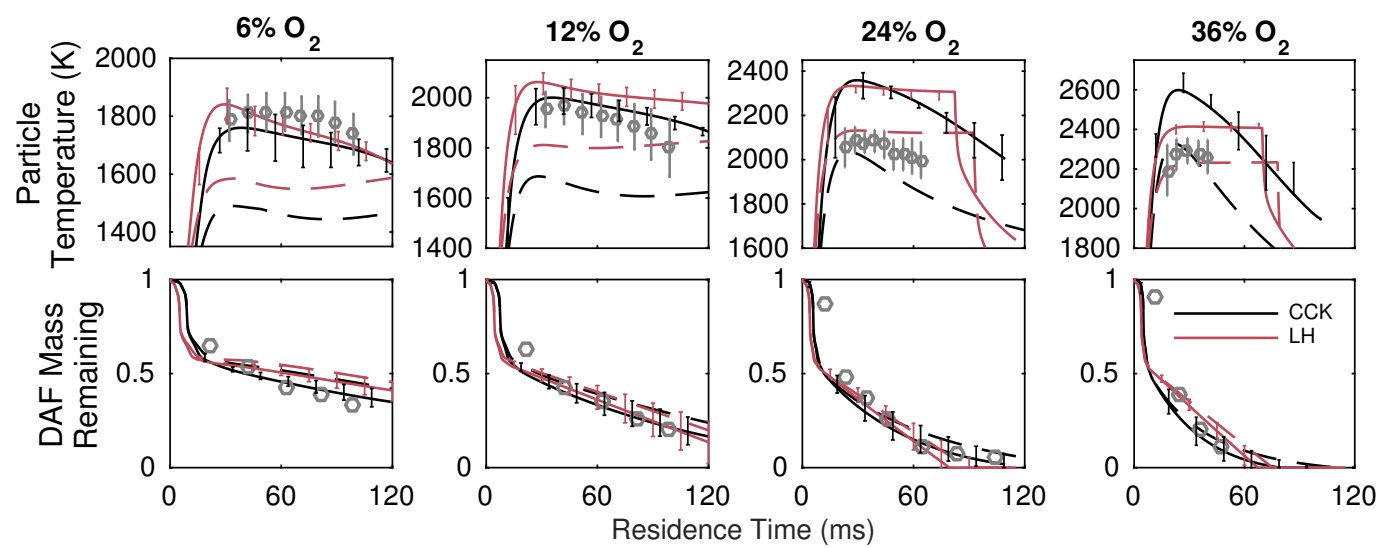

Figure 2: Predicted $T_{p}$ history and dry ash-free (DAF) mass fraction remaining for Eastern Bituminous coal with detailed kinetics (solid lines), flame-sheet (dashed lines) gas chemistry models, CCK and LH char chemistry models and CPD for devolatilization. Vertical bars coming off mass and temperature calculations resulting from detailed gas kinetics indicate the range of values obtained for initial particle diameters ranging from 106-125 $\mu \mathrm{m}$. Experimental data are represented by open circles with vertical bars indicating one standard deviation of $T_{p}$.

Over-prediction of particle temperatures may indicate that the value of $\alpha$ (see (3) ) is too small for $24 \%$ and $36 \%$ $\mathrm{O}_{2}$ cases considered, although the authors are unaware of any theoretical basis for choosing $\alpha$. Decreasing $\alpha$ from 0.3 to 0 results in a $T_{p}$ increase of up to $200 \mathrm{~K}$ for $6 \%$ and $12 \% \mathrm{O}_{2}$. However, the particle mass loss was not strongly affected by choosing a smaller value for $\alpha$. This observation indicates that a higher value of $\alpha$ may be appropriate for elevated $\mathrm{O}_{2}$ concentrations and further suggests that the char consumption rate is diffusion limited.

Despite the disagreement in $T_{p}$ prediction, mass loss predictions for the CCK and LH models match experimental 
data reasonably well. Figure 2 indicates that CCK tends to represent the mass loss data more accurately than LH. Both char models over-predict mass loss at early residence times under all $\mathrm{O}_{2}$ environments considered, with $\mathrm{LH}$ over-predicting mass-loss relative to CCK. This is due to an earlier onset of char oxidation predicted by LH which results in higher calculated particle heating and devolatilization rates. These differences are due primarily to the fact that the activation energies for oxidation used in LH are three orders of magnitude lower than those used in CCK.

Figure 2 also shows that the gas-phase kinetics have little effect on the predicted mass loss. Values for calculated DAF mass were slightly higher when using the flame-sheet model over detailed kinetics under all oxygen concentrations considered as a result of lower concentration of $\mathrm{O}_{2}$ near the particle at early residence times.

Figure 3 shows $T_{p}$ and mass histories for Highvale coal using LH and CCK paired with CPD for devolatilization and detailed gas kinetics. The ability to predict $T_{p}$ for the Highvale coal for both char models is similar to that for the Eastern Bituminous coal. $T_{p}$ calculations using both CCK and LH models are closer to experimentally observed values at $6 \%$ and $12 \% \mathrm{O}_{2}$ than those at either $24 \%$ or $36 \% \mathrm{O}_{2}$. Unlike the Eastern Bituminous coal, mass loss predictions by either char model do not closely match experimental data at initial $\mathrm{O}_{2}$ mole fractions greater than $12 \%$. It is presently unclear why this is.

In general, the LH model as implemented here does not fit the data as well as the implementation in [12]. This is especially true for particle temperature calculations. This may be due to the fact that the calculations performed in [12] use a single film model, and don't otherwise spatially resolve, species transport. It is not clear whether gas phase reactions are considered for char burnout calculations done in [12].

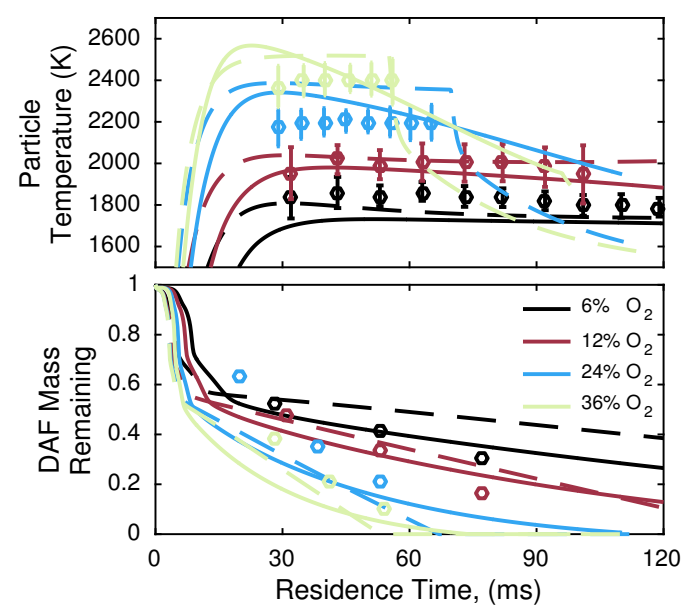

Figure 3: Experimental observations (circles) as well as predicted $T_{p}$ (top) and DAF mass fraction (bottom) remaining for the Highvale coal for detailed kinetics (solid lines) and flame-sheet (dotted lines) using CPD for devolatilization and CCK for char.

As demonstrated by Figure 2, $T_{p}$ is strongly influenced by the gas phase kinetics model, with detailed kinetics providing better agreement with experimental values then the flame-sheet model when either char reaction model was in use at $6 \%$ and $12 \% \mathrm{O}_{2}$. However, predictions resulting from the flame-sheet model tend to be closer at $24 \%$ and $36 \% \mathrm{O}_{2}$. In all instances, the flame-sheet model gives a $T_{p} 200-400 \mathrm{~K}$ lower than detailed kinetics. This is largely due to a lower calculated oxygen concentration near the particle (and thus, lower oxidation rates) at early residence times for the flame-sheet cases resulting from the "mixed is burnt" assumption.

Figure 4 compares calculated particle temperature, DAF mass remaining, volatile mass, and char oxidation rates 


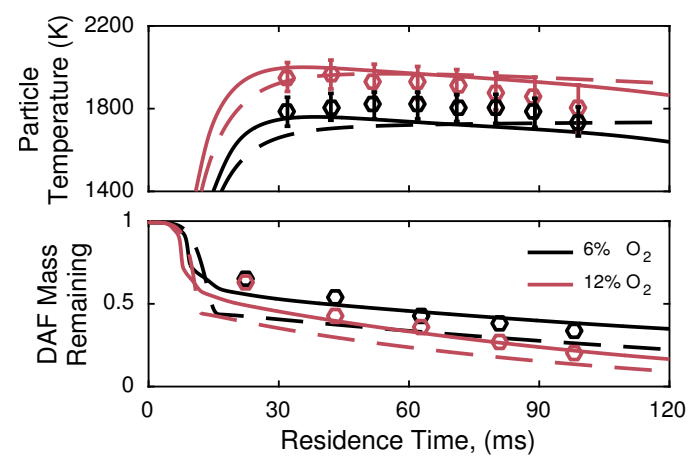

Figure 4: Experimental observations (circles) as well as predicted $T_{p}$ (top) and DAF mass fraction remaining (bottom) for Eastern Bituminous coal with CPD (solid lines) and KS (dotted lines) using detailed gas kinetics and CCK.
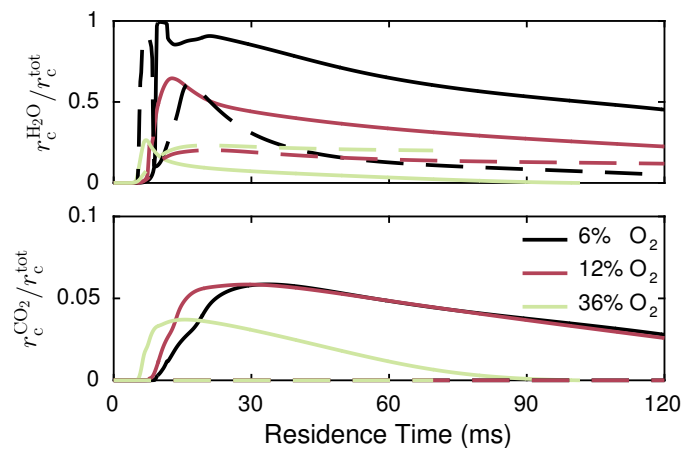

Figure 5: Predicted rates of gasification by $\mathrm{H}_{2} \mathrm{O}$ (top) and $\mathrm{CO}_{2}$ (bottom) normalized by the total rate of char consumption for Eastern Bituminous cases using detailed gas kinetics with CPD and CCK (solid lines) and LH (dashed lines).

for $6 \%$ and $12 \% \mathrm{O}_{2}$ environments using $\mathrm{CPD}$ and $\mathrm{KS}$ for devolatilization, and indicates that the devolatilization model has a noticeable influence on particle mass histories. For both $6 \%$ and $12 \% \mathrm{O}_{2}$, KS produces a significantly higher fraction of mass loss after devolatilization is completed. This occurs because the KS model predicts that a fraction of the fixed carbon as determined by ASTM proximate analysis devolatilizes at elevated temperatures. In any case, Figure 4 suggests that the chosen devolatilization model may have a substantial influence on char burnout calculations.

Figure 5 presents calculations for gasification by $\mathrm{CO}_{2}$ and $\mathrm{H}_{2} \mathrm{O}$ using $\mathrm{CCK}$ and $\mathrm{LH}$, and indicates that the two models have significantly different gasification behavior. CCK predicts that $\mathrm{H}_{2} \mathrm{O}$ gasification accounts for nearly all of char consumption in the $6 \% \mathrm{O}_{2}$ environment and 19-64\% for $12 \% \mathrm{O}_{2}$. The first order Arrhenius gasification model used in conjunction with the LH oxidation model predicts lower contributions from steam gasification: 7-60\% for $6 \%$ $\mathrm{O}_{2}$ and $7-21 \%$ for $12 \% \mathrm{O}_{2}$. These results indicate that a significant portion of the char consumption may be due to gasification by $\mathrm{H}_{2} \mathrm{O}$. Results presented in [10] also suggest that gasification reactions may contribute appreciably to the rate of char consumption in oxidative conditions. The char consumption from $\mathrm{CO}_{2}$ gasification is non-negligible, but is not nearly as appreciable as steam gasification.

\section{Conclusion}

This work considered two char and gas phase chemistry models along with two devolatilization models, each having different levels of complexity. Calculations from each combination of particle and gas phase model were compared 
to experimentally measured particle temperature and mass. Two coal types were considered under four different gas compositions. The results show that both the LH and CCK models provide trends for particle temperature that match the experimental data reasonably well for $6 \%$ and $12 \% \mathrm{O}_{2}$ if paired with detailed kinetics in the gas phase. However, neither of char chemistry models considered were able to accurately represent experimental values for particle temperature at elevated $\mathrm{O}_{2}$ concentrations. In general, particle mass predictions resulting from CCK more accurately represented data than those from LH. Executing the CCK model takes approximately 12 times longer than what is required for the LH model in the absence of gas phase calculations. Nonetheless, it is recommended that CCK is used in place of LH if accuracy of char burnout calculations is required. The comparison provided shows that use of the flame-sheet model results in particle temperatures that are significantly lower compared to cases where detailed chemistry is implemented. Calculated DAF mass remaining was similar for both detailed kinetics and infinitely-fast chemistry at $\mathrm{O}_{2}$ mole fractions of $12 \%$ and higher, which suggests that char burnout is insensitive to the choice of gas phase chemistry model for elevated oxygen concentrations.

Comparison of $\mathrm{CPD}$ and KS indicates that the devolatilization model has a noticeable impact on the particle mass loss predictions. When using KS, the rate of char depletion during devolatilization was, at times, greater than 3 times the char consumption rate when using CPD, resulting in significantly greater mass loss with KS than for CPD. Despite the increased rate of char consumption mid-devolatilization, the KS model predicts particle temperatures close to values predicted by CPD. This result is due to lower calculated peak gas temperatures when KS is used over CPD. Use of CPD is recommended over KS because particle mass calculations are closer to experimental values when using CPD.

Use of detailed gas chemistry produced only slightly more accurate mass loss predictions than the flame-sheet model. Particle temperature predictions were more accurate when using detailed kinetics than flame-sheet for the $6 \%$ and $12 \% \mathrm{O}_{2}$, but the converse is true for the two highest oxygen concentrations considered. Furthermore, the run times for simulations using detailed chemistry were about 10 times greater than simulations implementing the flamesheet model. All things considered, the flame-sheet model is recommended over detailed kinetics. The runtime for CPD is 11 times that of the KS model in the absence of gas phase calculations Despite this, use of CPD is suggested in place of KS given that CPD results in particle mass calculations that are much more accurate than calculations resulting from KS. From the results presented, it appears that in order to accurately predict particle mass loss, both devolatilization and char consumption need to be modeled with high fidelity, however the choice of gas phase chemistry has little impact. Accurate particle temperature measurements require modeling gas phase chemistry for $\mathrm{O}_{2}$ concentrations $\leq 12 \%$, but this is not the case for elevated $\mathrm{O}_{2}$.

Both char models indicate that steam gasification contributes significantly to the overall char consumption rate, especially at early residence times. Calculations suggest that gasification by $\mathrm{CO}_{2}$ accounts for less than $10 \%$ of the char consumption in all of the cases considered. These conclusions persist over a sizable range of bulk $\mathrm{O}_{2}$ concentrations.

\section{Acknowledgments}

This material is based upon work supported by the Department of Energy, National Nuclear Security Administration, under Award Number(s) DE-NA0002375. 
[1] H. Watanabe, M. Otaka, Fuel 85 (12-13) (2006) 1935-1943.

[2] S. Niksa, G. S. Liu, R. H. Hurt, Prog. Energy Combust. Sci. 29 (5) (2003) 425-477.

[3] G. S. Liu, S. Niksa, Prog. Energy Combust. Sci. 30 (6) (2004) 679-717.

[4] R. Hurt, J.-K. Sun, M. Lunden, A Kinetic Model of Carbon Burnout in Pulverized Coal Combustion, Combust. Flame 113 (1-2) (1998) 181-197.

[5] R. C. Shurtz, Ph.D. thesis, Brigham Young University.

[6] R. C. Shurtz, T. H. Fletcher, Energy \& Fuels 27 (6) (2013) 3022-3038.

[7] C. Gonzalo-Tirado, S. Jiménez, R. Johansson, J. Ballester, Combust. Flame 161 (4) (2014) 1085-1095.

[8] E. S. Hecht, C. R. Shaddix, J. S. Lighty, Combust. Flame 160 (8) (2013) 1499-1509.

[9] B. Goshayeshi, J. C. Sutherland, Combust. Flame 161 (7) (2014) 1900-1910.

[10] B. Goshayeshi, J. C. Sutherland, Proc. Combust. Inst. 35 (3) (2015) 2829-2837.

[11] N. Slavinskaya, M. Braun-Unkhoff, P. Frank, J. Eng. Gas Turbines Power 130 (2) (2008) 021504.

[12] J. J. Murphy, C. R. Shaddix, Combust. Flame 144 (4) (2006) 710-729.

[13] H. C. Hottel, A. F. Sarofim, Heat Transfer, Hemisphere Publishing, 1978.

[14] Q. M. Brewster, Thermal Radiative Transfer and Properties, John Wiley \& Sons, 1992.

[15] A. L. Brown, T. H. Fletcher, Energy and Fuels 4 (12) (1997) 745-757.

[16] T. H. Fletcher, A. R. Kerstein, R. J. Pugmire, M. Solum, D. M. Grant, A Chemical Percolation Model For Devolatilization: Summary, Tech. rep.

[17] H. Kobayashi, J. Howard, A. Sarofim, Symp. (Int.) Combust. 16 (1) (1977) 411-425.

[18] S. K. Ubhayakar, D. B. Stickler, C. W. Von Rosenburg, R. Gannon, Symp. (Int.) Combust. 16 (1) (1977) $427-436$.

[19] S. K. Bhatia, D. D. Perlmutter, AIChE J. 26 (3) (1980) 379-386.

[20] R. E. Mitchell, Symp. (Int.) Combust. 23 (1) (1991) 1297-1304.

[21] L. Tognotti, J. Longwell, A. Sarofim, Symp. (Int.) Combust. 23 (1) (1991) 1207-1213.

[22] A. D. Lewis, T. M. Holland, N. R. Marchant, E. G. Fletcher, D. J. Henley, E. G. Fuller, T. H. Fletcher, Energy \& Fuels 29 (3) (2015) 1479-1493.

[23] J. C. Sutherland, C. A. Kennedy, J. Comput. Phys. 191 (2) (2003) 502-524.

[24] J. Sutherland, N. Punati, A. R. Kerstein, A unified approach to the various formulations of the one-dimensional-turbulence model, Tech. rep., Institute for Clean Secure Energy (2010).

[25] I. Glassman, R. A. Yetter, N. G. Glumac, Combustion, Academic Press, 2014. 


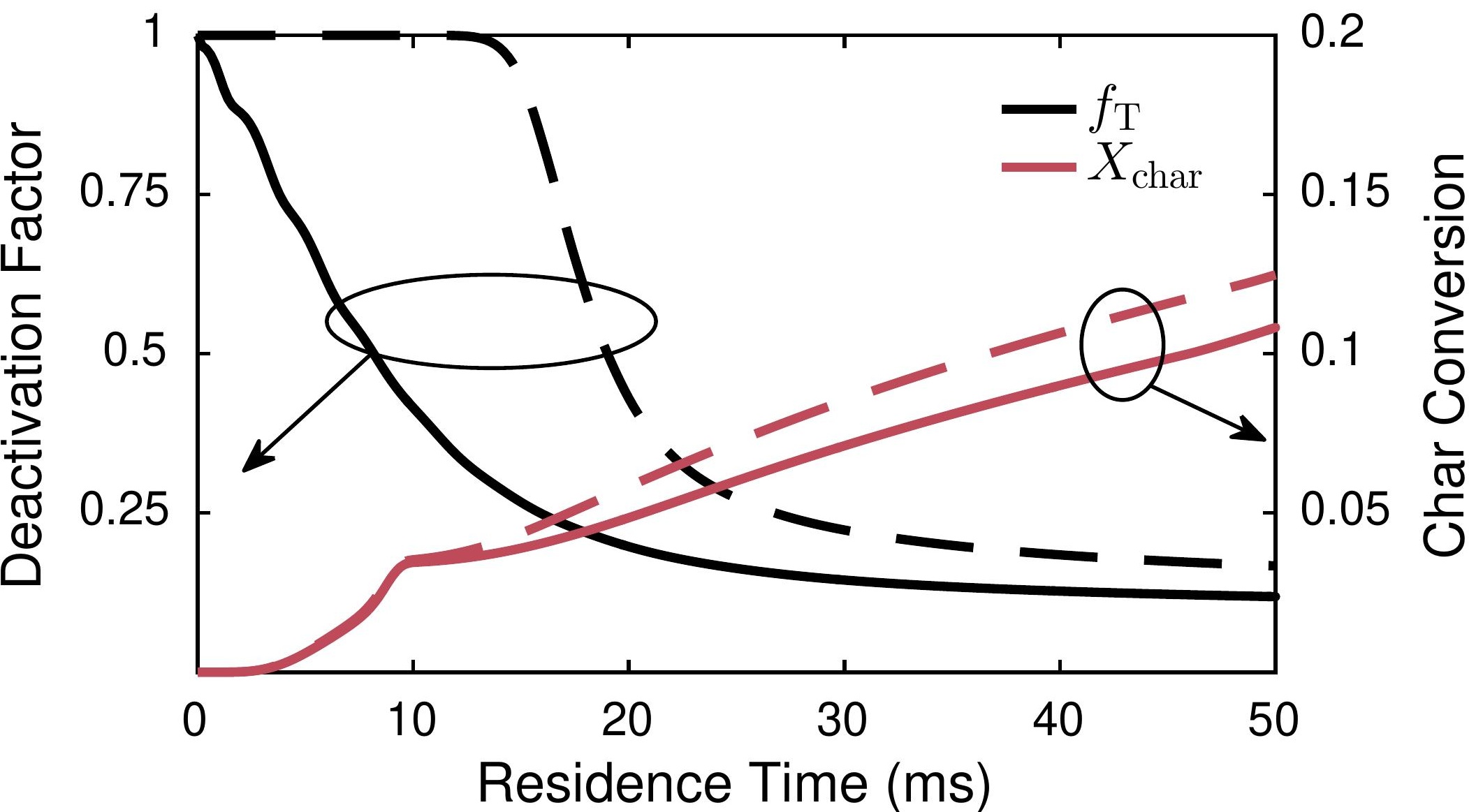




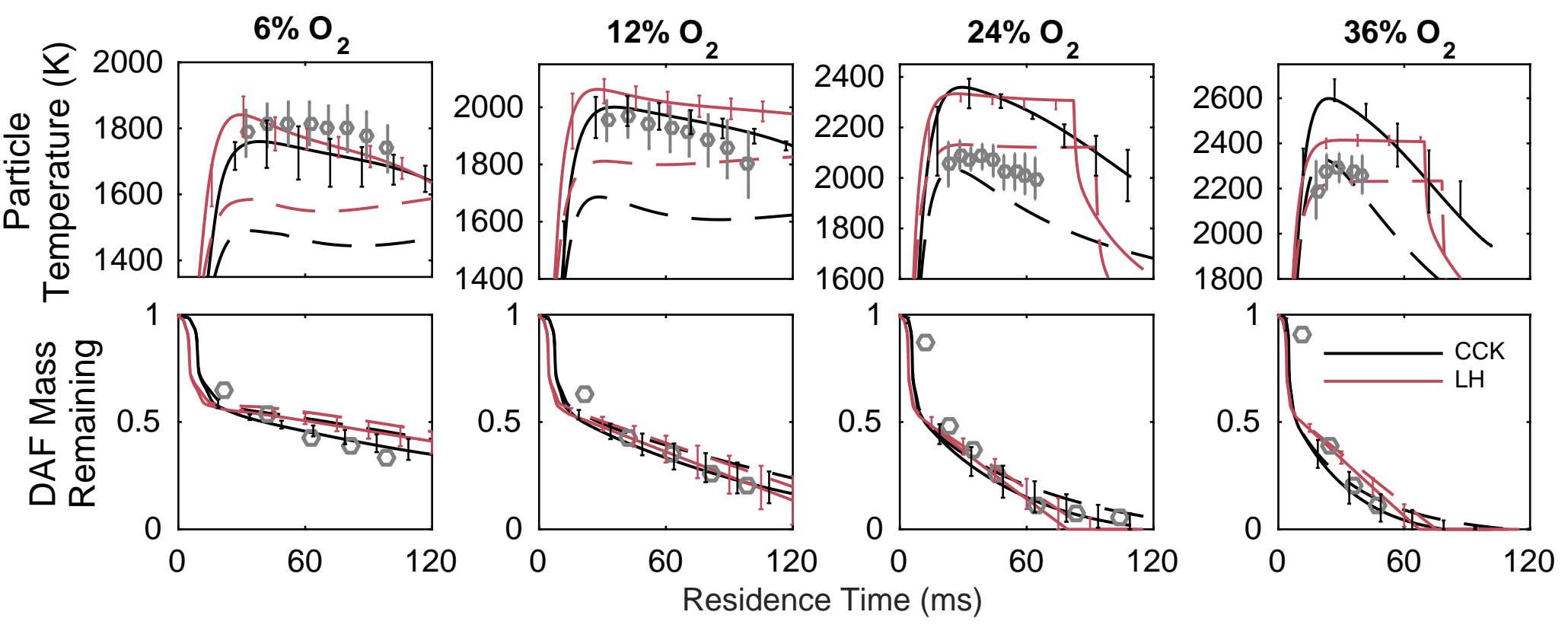




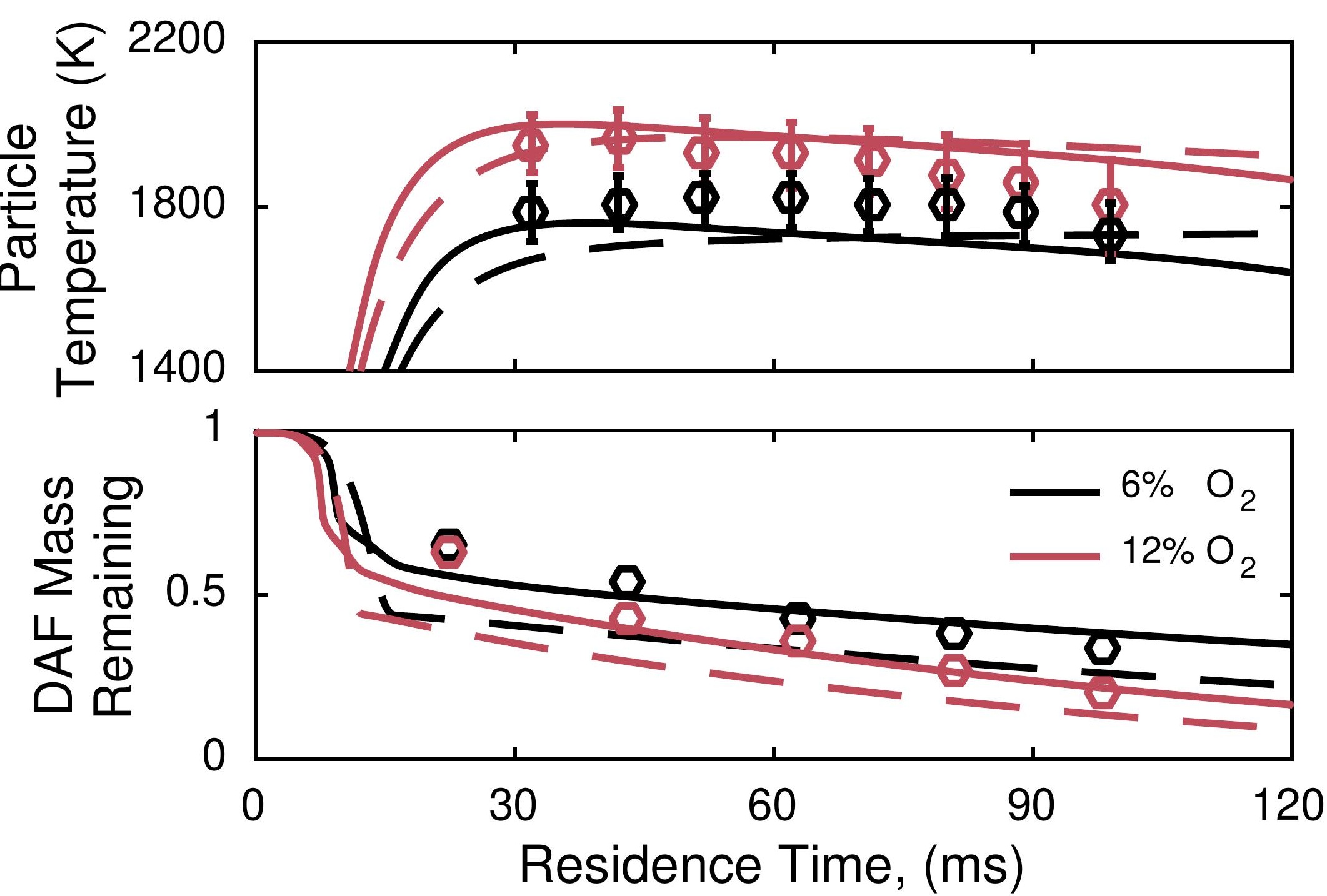



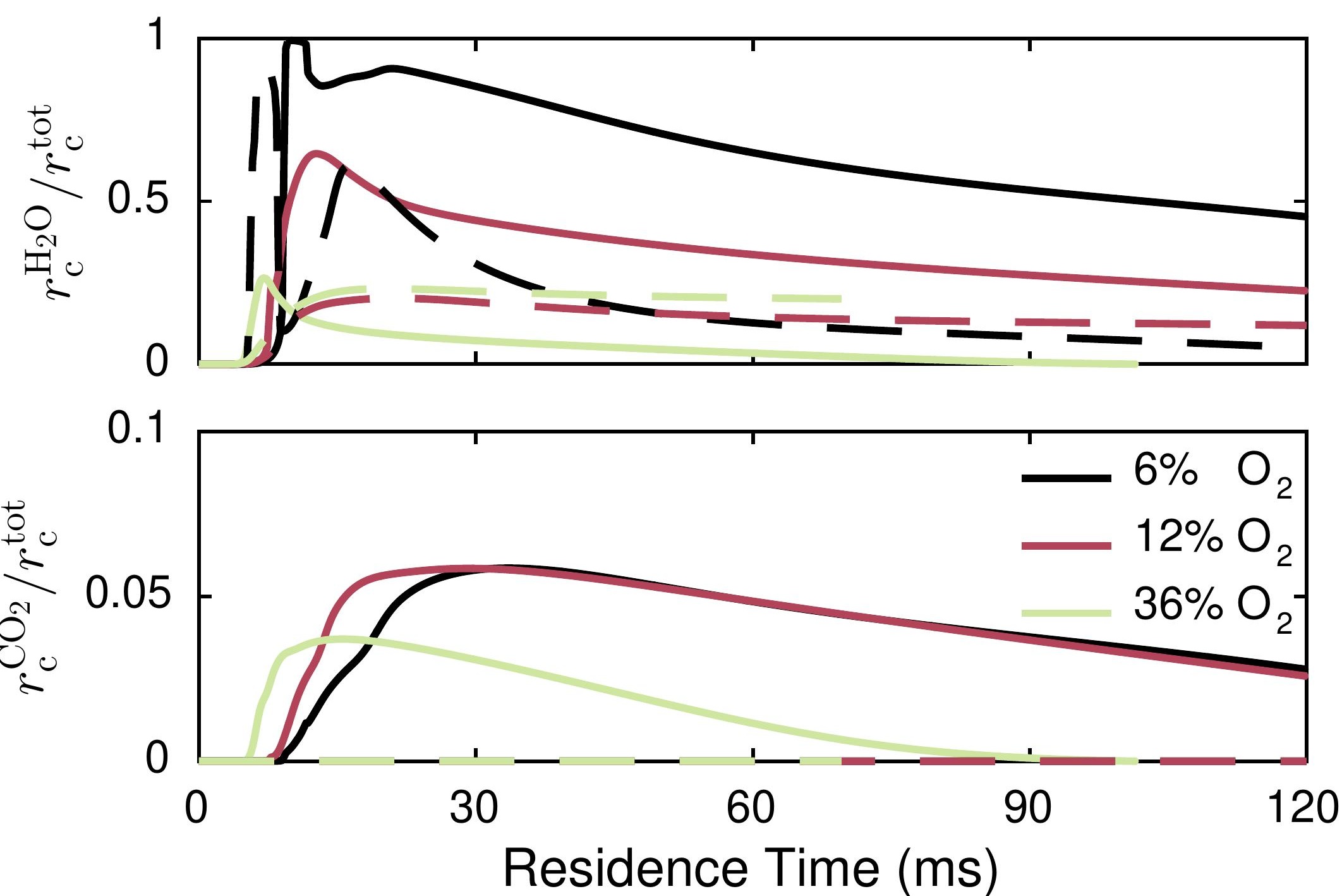

\section{Residence Time (ms)}

